\title{
ARTICLE
}

Received 14 Feb 2013 | Accepted 30 May 2013 | Published 28 Jun 2013

DOI: $10.1038 /$ ncomms3073

\section{Valley spin polarization by using the extraordinary Rashba effect on silicon}

Kazuyuki Sakamoto1, Tae-Hwan Kim², Takuya Kuzumaki', Beate Müller', Yuta Yamamoto', Minoru Ohtaka', Jacek R. Osiecki ${ }^{3}$, Koji Miyamoto ${ }^{4}$, Yasuo Takeichi ${ }^{5}$, Ayumi Harasawa ${ }^{5}$, Sebastian D. Stolwijk ${ }^{6}$, Anke B. Schmidt ${ }^{6}$, Jun Fujii ${ }^{7}$, R.I.G. Uhrberg ${ }^{3}$, Markus Donath ${ }^{6}$, Han Woong Yeom ${ }^{8}$ \& Tatsuki Oda ${ }^{9}$

The addition of the valley degree of freedom to a two-dimensional spin-polarized electronic system provides the opportunity to multiply the functionality of next-generation devices. So far, however, such devices have not been realized due to the difficulty to polarize the valleys, which is an indispensable step to activate this degree of freedom. Here we show the formation of $100 \%$ spin-polarized valleys by a simple and easy way using the Rashba effect on a system with $C_{3}$ symmetry. This polarization, which is much higher than those in ordinary Rashba systems, results in the valleys acting as filters that can suppress the backscattering of spin-charge. The present system is formed on a silicon substrate, and therefore opens a new avenue towards the realization of silicon spintronic devices with high efficiency.

\footnotetext{
${ }^{1}$ Department of Nanomaterials Science, Chiba University, Chiba 263-8522, Japan. ${ }^{2}$ Department of Physics and Center for Low Dimensional Electronic Symmetry, Pohang University of Science and Technology, Pohang 790-784, Korea. ${ }^{3}$ Department of Physics, Chemistry and Biology, Linköping University, Linköping S-581 83, Sweden. ${ }^{4}$ Hiroshima Synchrotron Radiation Centre, Hiroshima University, Higashi-Hiroshima 739-0046, Japan. ${ }^{5}$ Institute for Solid State Physics, The University of Tokyo, Chiba 277-8581, Japan. ${ }^{6}$ Physikalisches Institut, Westfälische Wilhelms-Universität Münster, Münster 48149, Germany. ${ }^{7}$ TASC Laboratory, IOM-CNR, SS 14, km 163.5, Trieste 34149, Italy. ${ }^{8}$ Center for Artificial Low Dimensional Electronic Systems of the Institute for Basic Science and Department of Physics, Pohang University of Science and Technology, Pohang 790-784, Korea. ${ }^{9}$ Institute of Science and Engineering, Kanazawa University, Kanazawa 920-1192, Japan.

Correspondence and requests for materials should be addressed to K.S. (email: kazuyuki_sakamoto@faculty.chiba-u.jp).
} 
S pintronic devices ${ }^{1-7}$, a new scheme of electronics using the spin degrees of freedom together with the electron charge, can be classified into two categories, the magnetic spintronic devices for information storage, and semiconductor ones such as the spin field-effect transistor ${ }^{1}$ for logical operations. Of these two categories, the realization of semiconductor spintronic devices is far behind the magnetic ones, which already appear in the market as, for example, read heads of hard disk drives. The main reason is the difficulty to handle the spin current, the key principle for operating semiconductor spintronic devices. In nonmagnetic materials, spin degeneracy is lifted by spin-orbit coupling in combination with space inversion asymmetry, such as at solid surfaces and interfaces, that is, the socalled Rashba effect ${ }^{8,9}$. Ordinarily, the Rashba effect produces a pair of spin-polarized bands in $\mathbf{k}$ space with the spin polarization vector in the surface plane and a Fermi surface consisting of two concentric circles with opposite chiral spin textures. As shown in Fig. 1a, the presence of these two Fermi circles allows electrons to backscatter, and thus can greatly reduce the efficiency of the spin transport. Topological insulators with a single Dirac cone $e^{10}$, a new state of matter, show a single circle with chiral spin texture (Fig. 1b) that would prevent backscattering of electrons in the opposite direction provided the spin is $100 \%$ polarized and there is no influence of magnetic impurities. However, owing to the actual spin polarization that is far from $100 \%$ (ref. 11) and the tilting of the spin direction ${ }^{12}$, electron spins can be backscattered in this material as well ${ }^{13}$. Consequently, the complete suppression of backscattering, an essential requirement to achieve high performance semiconductor spintronic devices ${ }^{13}$, is still an unresolved issue. In this respect, a very promising approach is combining valley physics, which restricts the scattering direction as in the case of graphene $\mathrm{e}^{14,15}$ (Fig. 1c), with two-dimensional spin-polarized electronic systems. It is also crucial to spin-polarize the valleys, as shown in Fig. 1d, which provides the filtering function that suppresses the probability of backscattering. Furthermore, previous propositions for valley polarization in graphene require difficult ways of electron confinement. In contrast, we propose a simple and easy method.

Here, we show that a $\mathrm{Si}(111)$ surface with slightly more than one monolayer (ML) of $\mathrm{Tl}$ on top forms $100 \%$ spin-polarized valley surface states for which the backscattering can be tuned to disappear. The heavy element $\mathrm{Tl}$ gives rise to the strong spinorbit coupling, which induces the large spin splitting responsible for the spin-polarized valleys, and the use of $\mathrm{Si}$ as substrate will issue a guideline to the realization of silicon spintronic devices $^{16-18}$. The atomic structure of the Tl/Si(111)- $(1 \times 1)$ surface at a coverage of $1 \mathrm{ML}$ and the corresponding surface Brillouin zone (SBZ) are displayed in Fig. 2a,b. Tl atoms are adsorbed on the 3 -fold hollow site (the $T_{4}$ site) of a $\mathrm{Si}(111)$ $(1 \times 1)$ ideal surface, leading to a 3 -fold symmetric structure with mirror planes in the [112] and the two other corresponding directions. This surface structure leads to the $\overline{\mathrm{K}}$ (and $\overline{\mathrm{K}^{\prime}}$ ) points to have $C_{3}$ symmetry, which lifts the spin degeneracy at these points and forces the polarization vector of the fully polarized spin to be perpendicular to the surface ${ }^{19-21}$. This differs from that of normal Rashba systems, where the polarization is parallel to the surface and perpendicular to the wave vector (Fig. 1a). The out-of-plane spins of the occupied surface state, which were observed experimentally with high-resolution angle-resolved photoelectron spectroscopy (ARPES) ${ }^{20}$ and discussed theoretically ${ }^{19,21}$, are unfortunately far below the Fermi level and will therefore hardly contribute to any charge transport.

\section{Results}

Electronic structure of undoped $\mathrm{Tl} / \mathrm{Si}(111)$. As displayed in Fig. 2c, theoretical calculations predict the presence of an unoccupied surface electronic band with a substantially larger spin splitting just above the Fermi level at the $\overline{\mathrm{K}}$ point. This suggests a possibility to make a metallic spin-polarized band by doping electrons into the $\mathrm{Tl} / \mathrm{Si}(111)-(1 \times 1)$ surface while keeping the substrate semiconducting as there are no bulk continuum states at the corresponding energies. In Fig. 3a, we show the occupied electronic band of an undoped $\mathrm{Tl} / \mathrm{Si}(111)-(1 \times 1)$ surface obtained by ARPES and the unoccupied band obtained by spinand angle-resolved inverse photoemission spectroscopy (SARIPES) along the $k_{\mathrm{x}}$ direction in Fig. 3b. The Rashba splitting is clearly observed in the occupied state, and two spin-polarized surface bands are observed above the Fermi level. The polarization vectors of these unoccupied surface bands are perpendicular to the surface and the spins are $100 \%$ polarized as in the case of the occupied states at the $\overline{\mathrm{K}}$ point. This experimental finding agrees well with the polarization vector predicted in the theoretical calculation.

Electronic structure of doped $\mathrm{Tl} / \mathrm{Si}(111)$. In this study, we doped the $\mathrm{Tl} / \mathrm{Si}(111)-(1 \times 1)$ surface by adsorbing a small amount of extra $\mathrm{Tl}$. The extra $\mathrm{Tl}$ does not change the low-energy electron diffraction pattern for $\mathrm{Tl}$ coverage of 1.00-1.12 ML (Fig. 3c). a

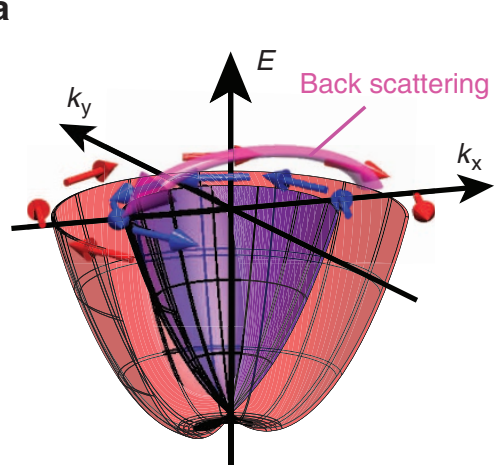

b

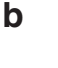

No back scattering in $100 \%$ spin-polarized system
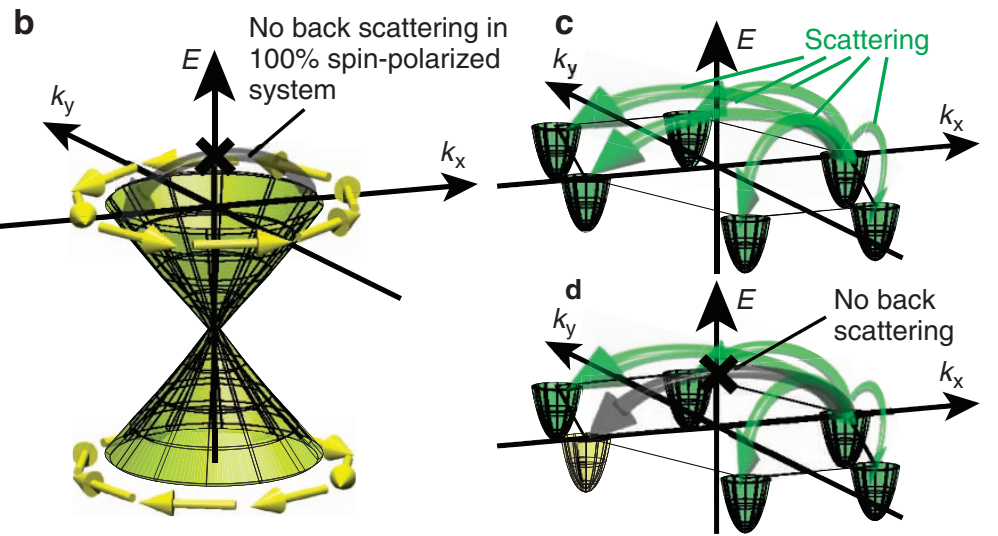

Figure 1 | Dispersion and spin texture of spin-polarized bands and the valley scattering. Spin-polarized bands of (a) an ordinary Rashba system and (b) topological insulator. (c) A system with non-polarized electron valleys at the $\bar{K}$ and $\overline{K^{\prime}}$ points of the SBZ. In this case, scattering is limited to the $\bar{K}-\bar{K}\left(\overline{K^{\prime}}\right)$ directions, which is in contrast to the case of systems with Fermi circles at the $\bar{\Gamma}$ point where electrons scatter in all directions. (d) A system in which one valley, the one indicated by yellow, is polarized. Electrons cannot backscatter in this case. 
As shown in Fig. 3d, a state appears at the Fermi level with an extra Tl coverage of $0.01 \mathrm{ML}$, and it moves continuously downward in energy by adding more and more Tl. This agrees with the appearance of a metallic property with extra $\mathrm{Tl}$ in former studies $^{22,23}$. This result demonstrates that electrons are doped into the unoccupied surface state. To quantitatively discuss the observed metallic band obtained at $\mathrm{Tl}$ coverages of 1.12 and 1.04 ML, we show close ups of the band in Fig. $3 \mathrm{e}$ and $\mathrm{f}$, respectively. The red dots in Fig. $3 e$ mark the peak positions of the metallic band extracted from the ARPES spectra, and the

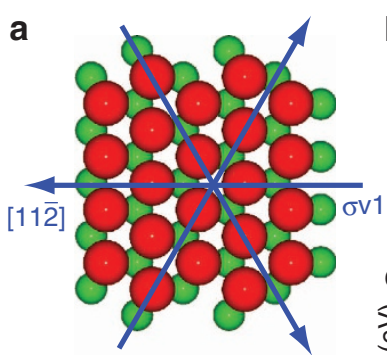

Top view

Side view

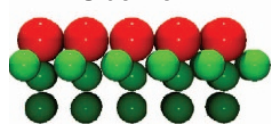

b

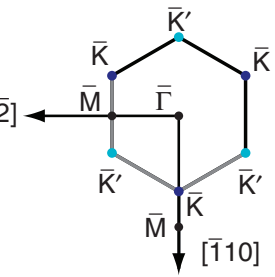

c

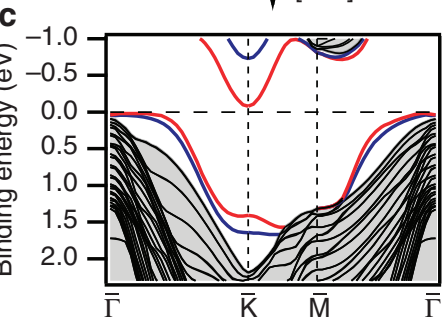

Figure 2 | Basic properties of TI/Si(111)-(1 $\times \mathbf{1 ) .}(\mathbf{a})$ Atomic structure of the system. Red and green circles represent the $\mathrm{TI}$ and Si atoms, respectively, and the blue arrows indicate the mirror planes. (b) The SBZ of TI/Si(111)-(1 $\times 1)$. The symmetry points $\overline{\mathrm{K}}$ and $\overline{\mathrm{K}^{\prime}}$ are indicated by dark-blue and light-blue circles. (c) Energy-band dispersions obtained by a relativistic first-principles band calculation. Red and blue lines are spin-polarized surface states. yellow line represents the dispersion obtained by fitting the peak positions using a parabola. The result indicates that the bottom of the metallic band is located at a binding energy $\left(E_{\mathrm{B}}\right)$ of $65 \mathrm{meV}$, and that the effective mass is $\sim 0.35 \mathrm{~m}_{\mathrm{e}}$ along the $k_{\mathrm{x}}$ direction at 1.12 ML. At 1.04 ML, the bottom of the metallic band is shifted to $E_{\mathrm{B}}=45 \mathrm{meV}$, but the effective mass is the same. This agrees with a rigid-shift picture of the spin-polarized unoccupied surface band by electron doping. Furthermore, in the light of the same effective mass along the $k_{\mathrm{y}}$ direction and the roughly circular shape of the Fermi surface shown in Fig. $3 g$, we conclude that the spin-polarized metallic band of the $\mathrm{Tl} / \mathrm{Si}(111)-(1 \times 1)$ surface forms valleys with a parabolic shape at the $\overline{\mathrm{K}}$ and $\overline{\mathrm{K}^{\prime}}$ points.

Spin-polarized valleys. To grasp the overall character of the metallic band, the spin-resolved ARPES (SARPES) spectra measured around the $\overline{\mathrm{K}}$ and $\overline{\mathrm{K}^{\prime}}$ points along the $k_{\mathrm{y}}$ direction, and those around the $\overline{\mathrm{K}}$ point along the $k_{\mathrm{x}}$ direction are shown in Fig. 4a,b,c for a sample with Tl coverage of $1.12 \mathrm{ML}$. Here, the SARPES spectra were obtained with an experimental setup that is sensitive to spins with polarization vectors perpendicular to the surface ( $z$ direction). The red and blue spectra represent the spin pointing in the positive (spin-up) and the negative $z$ direction (spin-down) as shown in the inset. It is apparent from these spectra that the metallic band is $100 \%$ spin-up at the $\overline{\mathrm{K}}$ point, and $100 \%$ spin-down at the $\overline{\mathrm{K}^{\prime}}$ point. The correspondence between the spin directions of the metallic band and those of the occupied bands located at an $E_{\mathrm{B}}$ of about $2 \mathrm{eV}$ agrees well with the spin directions predicted theoretically (Fig. 2c). The time-reversal symmetry $[E(\mathbf{k}, \uparrow)=E(-\mathbf{k}, \downarrow)]$ is preserved in this system, but the inversion asymmetry lifts the spin degeneracy and leads to opposite spin directions at $\overline{\mathrm{K}}$ and $\overline{\mathrm{K}^{\prime}}$. Therefore, the metallic valleys are polarized intrinsically in this peculiar Rashba system.

Figure 4d shows schematically the spin-resolved electronic structure of a $\mathrm{Si}(111)$ surface with slightly more than $1 \mathrm{ML}$ of $\mathrm{Tl}$ on top. The green (purple) hexagon represents the SBZ, here drawn to indicate the position of the Fermi level with a $\mathrm{Tl}$ a

)

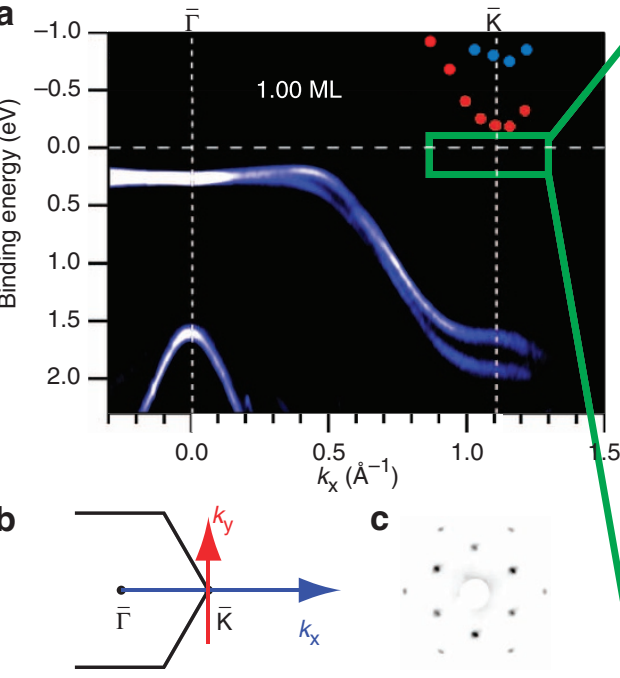

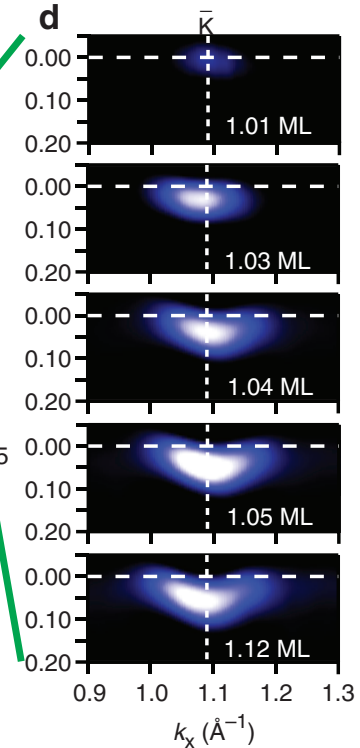
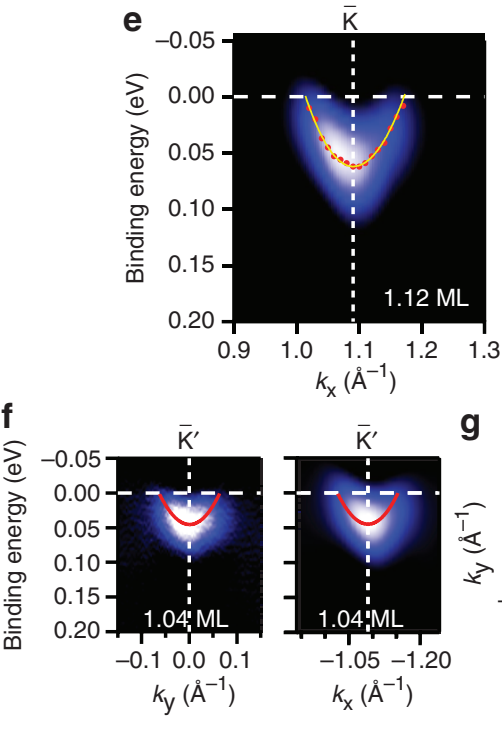

g

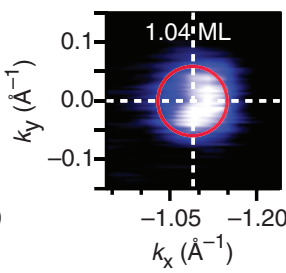

Figure 3 I Spin-polarized metallic band. (a) ARPES result together with the SARIPES one, which is indicated by red and blue circles of a Si(111) surface covered with $1.00 \mathrm{ML}$ of $\mathrm{TI}$. (b) Directions of $k_{\mathrm{x}}$ and $k_{\mathrm{y}}$ with respect to the SBZ. (c) Low-energy electron diffraction pattern of the TI/Si(111)-(1 $\times 1$ ) surface obtained at a TI coverage of $1.00 \mathrm{ML}$ with a primary electron energy of $111 \mathrm{eV}$. (d) Metallic band observed at the $\overline{\mathrm{K}}$ point for different $\mathrm{TI}$ coverages. The area in $\mathbf{d}$ corresponds to the area indicated by the green box in $\mathbf{a}$. Detailed band dispersion of the metallic band at a $\mathrm{TI}$ coverages of (e) $1.12 \mathrm{ML}$ along the $k_{\mathrm{x}}$ direction and (f) of 1.04 ML along the $k_{\mathrm{x}}$ and $k_{\mathrm{y}}$ directions. (g) Fermi surface of Si(111) covered with 1.04 ML of $\mathrm{TI}$. 

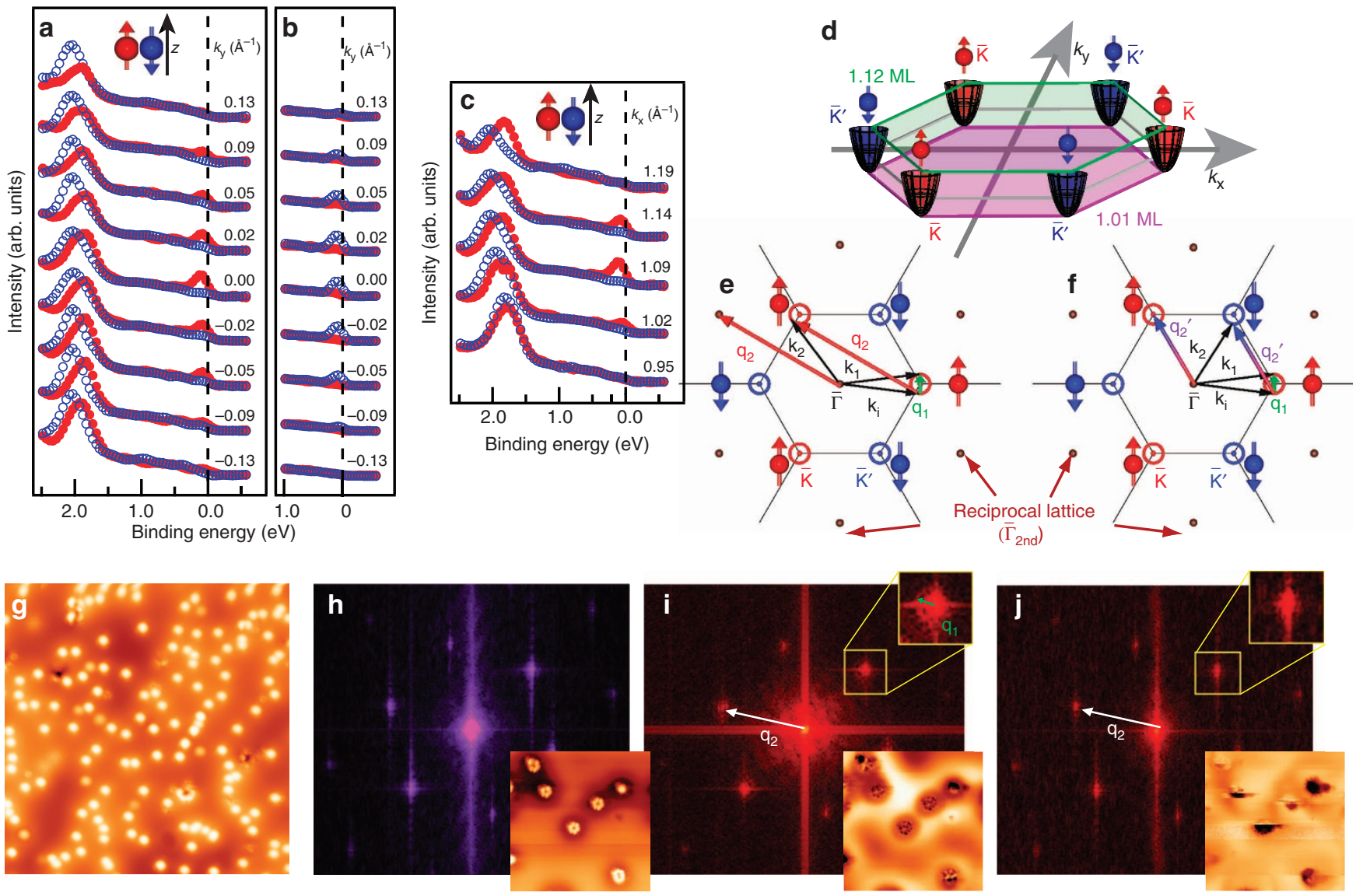

Figure 4 | Spin character of the metallic band. SARPES spectra measured along the $k_{y}$ direction around (a) the $\overline{\mathrm{K}}$ point and (b) the $\overline{\mathrm{K}^{\prime}}$ point, and (c) along the $k_{\mathrm{x}}$ direction around the $\overline{\mathrm{K}}$ point. The directions of the wave vectors $k_{\mathrm{x}}$ and $k_{\mathrm{y}}$ are shown in Figure $3 \mathrm{~b}$. The red (blue) spectra represent the spin pointing to positive (negative) $z$ as indicated in the inset. (d) Schematic illustration of the spin-polarized surface states at $\bar{K}$ and $\overline{K^{\prime}}$. The green (purple) hexagon represents the SBZ. The position of the SBZ is here used to indicate the Fermi level at a coverage of 1.12 (1.01) ML. Illustrations of scattering processes (e) without and (f) with spin flipping. $\mathbf{k}_{\mathbf{i}}$ is the wave vector of the incident electrons, and $\mathbf{k}_{\mathbf{1}}$ and $\mathbf{k}_{\mathbf{2}}$ are the wave vectors of the scattered ones. $\mathbf{q}_{\mathbf{1}}$ and $\mathbf{q}_{\mathbf{2}}\left(\mathbf{q}_{\mathbf{2}}{ }^{\prime}\right)$ are the intravalley and intervalley scattering wave vectors. (g) A $41.7 \times 41.7 \mathrm{~nm}^{2}$ STM image of a Si(111) surface with $1.01 \mathrm{ML}$ of adsorbed TI. $V_{\text {sample }}$ and tunneling current are $+1.0 \mathrm{~V}$ and $3.0 \mathrm{nA}$, respectively. (h) Two-dimensional FFT map of an STM topography obtained for a sample with $<0.0005 \mathrm{ML}$ of extra Tl at a $V_{\text {sample }}$ of $+0.25 \mathrm{~V}$. The inset in $\mathbf{h}$ is the $30 \times 30 \mathrm{~nm}^{2} \mathrm{STM}$ topography. $q$ space maps of scattering amplitudes obtained from FFT of $d / / d V$ maps at $V_{\text {sample }}$ of $(\mathbf{i})+0.5 \mathrm{~V}$ and $(\mathbf{j})+0.25 \mathrm{~V}$. The insets in $\mathbf{i}$ and $\mathbf{j}$ are the quasiparticle interference patterns of $30 \times 30 \mathrm{~nm}^{2}$ images.

coverage of 1.12 (1.01) ML. The Fermi level of the $1.12 \mathrm{ML} \mathrm{Tl}$ surface cuts through the parabolic metallic band, resulting in constant-energy contours at the Fermi level (the Fermi surface) as small circles with a radius of $\sim 0.1 \AA^{-1}$ centred at the $\overline{\mathrm{K}}$ and $\overline{\mathrm{K}^{\prime}}$ points. This Fermi surface resembles closely that of an electrondoped graphene single layer ${ }^{24}$, although the underlying dispersions and the spin properties are different. The graphene single layer is associated with a pseudo spin instead of real spin.

Scattering process. The Fermi surface and its spin structure govern the elastic electron scattering between states located in a single valley (intravalley scattering), and states located in two valleys at $\overline{\mathrm{K}}\left(\overline{\mathrm{K}^{\prime}}\right)$ points or one at $\overline{\mathrm{K}}$ and the other at $\overline{\mathrm{K}^{\prime}}$ (intervalley scattering). $\mathbf{k}_{\mathbf{i}}$ in Fig. $4 \mathrm{e}, \mathrm{f}$ denotes the wave vector of the incident state, and $\mathbf{k}_{\mathbf{1}}$ and $\mathbf{k}_{\mathbf{2}}$ are the wave vectors of the scattered states. Of the two dominant classes of scattering vectors, $\mathbf{q}_{\mathbf{1}}$ corresponds to intravalley scattering, and $\mathbf{q}_{\mathbf{2}}\left(\mathbf{q}_{\mathbf{2}}{ }^{\prime}\right)$ to intervalley scattering between two $\overline{\mathrm{K}}$ points or two $\overline{\mathrm{K}^{\prime}}$ points (between $\overline{\mathrm{K}}$ and $\overline{\mathrm{K}^{\prime}}$ ). The absence of intervalley scattering between $\overline{\mathrm{K}}$ and $\overline{\mathrm{K}^{\prime}}$ in Fig. $4 \mathrm{e}$ results from the fact that the spin at the $\overline{\mathrm{K}}$ valley is completely opposite to that at the $\overline{\mathrm{K}^{\prime}}$ valley so that the overlap of the scattered wave to the incident one is zero if the spin-flip does not occur as in Fig. 4f. This is different from the graphene case in which the intervalley scattering between $\overline{\mathrm{K}}$ and $\overline{\mathrm{K}}^{\prime}$ occurs due to the helical pseudo spin texture ${ }^{25-27}$.

To confirm the scattering process experimentally, we performed a scanning tunnelling microscope (STM) study of $\mathrm{Tl} / \mathrm{Si}(111)$ $(1 \times 1)$ surfaces with extra Tl. Figure $4 \mathrm{~g}$ shows a $41.7 \times 41.7 \mathrm{~nm}^{2}$ topographic STM image of a surface with $0.01 \mathrm{ML}$ of extra Tl. As shown in this figure, the apparent size of the extra $\mathrm{Tl}$ adsorbates (bright spots in the image) has a diameter of $\sim 1.3 \mathrm{~nm}$ that makes it difficult to observe the electronic structure of the bare $\mathrm{Tl} / \mathrm{Si}(111)$ $(1 \times 1)$ surface area. Thus, the study of the quasiparticle interference pattern, which is indispensable to understand the scattering process, was not possible at the smallest coverage showing a metallic band in ARPES. We therefore decreased the amount of extra $\mathrm{Tl}$ to $<0.0005 \mathrm{ML}$ and accessed the electron scattering at unoccupied states above the Fermi level. The rigid-shift model of the spinpolarized unoccupied surface band allows us to discuss the scattering process under this condition.

Figure $4 \mathrm{~h}$ displays a reciprocal lattice map obtained by a twodimensional fast Fourier transform of an STM topography image of the sample with $<0.0005 \mathrm{ML}$ of extra Tl. The six reciprocal lattice points located in the second Brillouin zones are clearly observed together with weak signals from reciprocal lattice points 
of the third Brillouin zones. (Note that the hexagon formed by the six reciprocal lattice points in Fig. 4h,i,j appears rotated compared with those shown in Fig. 4e,f. The orientation of the hexagons in Fig. 4h,i,j just reflects the actual orientation of the sample in the STM set up.) Quasiparticle interference patterns are observed in the $d I / d V$ mapping obtained at $V_{\text {sample }}=+0.5 \mathrm{~V}$ and $+0.25 \mathrm{~V}$ (inset of Fig. 4i,j). The $q$ space maps of scattering amplitudes obtained from fast Fourier transform of these $d I / d V$ maps are displayed in Fig. 4i,j. The bright spots in these figures appear at exactly the same position as those of the reciprocal lattice points. At $V_{\text {sample }}=+0.5 \mathrm{~V}$, which corresponds to an energy of $0.25 \mathrm{eV}$ above the bottom of the valley, both the intravalley scattering vectors $\mathbf{q}_{1}$ and the intervalley one $\mathbf{q}_{\mathbf{2}}$ are observed. Of these two vectors, the length of $\mathbf{q}_{1}, \sim 2.7 \mathrm{~nm}^{-1}$, agrees well with the diameter of the valley obtained experimentally by SARIPES and by theoretical calculations at the corresponding energy. As extra spots located at the $(\sqrt{3} \times \sqrt{3})$ positions (the positions of the $\overline{\mathrm{K}}$ and $\overline{\mathrm{K}^{\prime}}$ points) should appear in the case of spin-flip scattering just like the spots observed in graphene due to the scattering vector $\mathbf{q}_{2}{ }^{\prime}$ (refs 25-27), the results in Fig. 4i,j indicate that there is no spin-flip scattering in the present case.

\section{Discussion}

As shown in Fig. 4i,j, scattering in the diametrically opposite direction via $\mathbf{q}_{2}$ is restricted but scattering might occur via $\mathbf{q}_{1}$, and thus the backscattering probability is restrained but not zero at $V_{\text {sample }}=+0.5 \mathrm{~V}$. At $V_{\text {sample }}=+0.25 \mathrm{~V}$, that is, the scattering at the bottom of the valley, $\mathbf{q}_{1}$ becomes infinitely small and only $\mathbf{q}_{2}$ remains. As the spin polarization vector remains unchanged at the bottom of the valley, this indicates the absence of any scattering in the diametrically opposite direction. Note that in contrast to the present case, backscattering would be possible even at the bottom of the valley in normal Rashba systems with spin polarization parallel to the surface. The present study shows that the Rashba effect on a system with $C_{3}$ symmetry completely spin polarizes the valleys in an easy and simple way and makes them acting as filters that suppress the backscattering of spin carriers. Thus, combining peculiar Rashba spins with valleytronics opens up an avenue to greatly improve the efficiency of spin currents for silicon spintronics applications.

\section{Methods}

Sample preparation method. A Si(111) surface was cleaned by annealing up to $1,520 \mathrm{~K}$ for a few seconds. Tl was deposited onto the clean Si surface at $300 \mathrm{~K}$, followed by annealing at $500 \mathrm{~K}$ for a couple of minutes. The intensity of the Tl $5 \mathrm{~d}$ core level was monitored to obtain the $\mathrm{Tl}$ coverage.

ARPES measurements. All PES experiments were performed in ultrahigh vacuum chambers under a base pressure of $<5 \times 10^{-9} \mathrm{~Pa}$. High-resolution ARPES and SARPES measurements have been carried out at different laboratories. ARPES data have been obtained at synchrotron radiation facilities, beamline I4 at MAX-lab, Sweden and the APE beamline at Elettra, Italy, using photon energies from 15 to $40 \mathrm{eV}$, with energy and momentum resolutions of $10 \mathrm{meV}$ and below $1 \%$ of the SBZ, respectively. The sample was maintained at $100 \mathrm{~K}$ during the ARPES measurements. The SARPES experiments were performed at beamline $19 \mathrm{~A}$ at Photon Factory, High Energy Accelerator Research Organization, Japan, using a photon energy of $17 \mathrm{eV}$ at an energy resolution of $20 \mathrm{meV}$ and momentum resolution below $2 \%$ of the SBZ. A very-low-energy-electron-diffraction-type spin polarimeter ${ }^{28}$ was part of the data collection system.

SARIPES measurements. The experiments were performed at the University of Münster, Germany, in an ultrahigh vacuum apparatus (base pressure $<3 \times 10^{-9} \mathrm{~Pa}$ ) with a combined SARIPES and SARPES setup ${ }^{29}$. The SARIPES data were retrieved by using spin-polarized electrons with energy between 7 and $14 \mathrm{eV}$ emitted from a GaAs photocathode and detecting photons from the sample with a Geiger-Müller counter at a detection energy of $9.9 \mathrm{eV}$.

STM measurements. The STM experiments were performed in an ultrahigh vacuum cryogenic STM (Unisoku, Japan), equipped with a Nanonis controller
(Specs, Germany). The base pressure was $6 \times 10^{-9} \mathrm{~Pa}$, and the temperature was maintained at $78 \mathrm{~K}$. The topography and $d I / d V$ maps were simultaneously taken by a lock-in detection method with a modulation voltage of $10-20 \mathrm{mV}$ at $1-2 \mathrm{kHz}$ and a set point of $0.1-3 \mathrm{nA}$

Density functional theory calculation. Calculations were carried out by using the generalized gradient approximation ${ }^{30}$ in the Kohn-Sham theory ${ }^{31}$. The energy cutoffs of 25 and 300 Ry were used for wave functions and charge densities $^{32}$, and a repeated slab model was used for the surface calculation. The slab contains a Tl ML, $24 \mathrm{Si} \mathrm{ML}$ and $1 \mathrm{H} \mathrm{ML}$. The atomic positions, except those of the $\mathrm{H}$ atoms and the $\mathrm{Si}$ atoms bonded to $\mathrm{H}$, were fully optimized to an assumed criterion of atomic force $\left(<0.01 \mathrm{eV} \AA^{-1}\right)$. The slabs were separated by $9.7 \AA$ of vacuum

\section{References}

1. Datta, S. \& Das, B. Electronic analog of the electro-optic modulator. Appl. Phys. Lett. 56, 665-667 (1990).

2. Hägele, D., Oestreich, M., Rühle, W. W., Nestle, N. \& Eberi, K. Spin transport in GaAs. Appl. Phys. Lett. 73, 1580-1582 (1998).

3. Kikkawa, J. M. \& Awschalom, D. D. Lateral drag of spin coherence in gallium arsenide. Nature 397, 139-141 (1999).

4. Wolf, S. A. et al. Spintronics: a spin-based electronics vision for the future. Science 294, 1388-1495 (2001).

5. Zŭtić, I., Fabian, J. \& Das Sarma, S. Spintronics: fundamentals and applications Rev. Mod. Phys. 76, 323-410 (2004).

6. Awschalom, D. D. \& Flatté, M. E. Challenges for semiconductor spintronics. Nat. Phys. 3, 153-159 (2007)

7. Nadj-Perge, S., Frolov, S. M., Bakkers, E. P. A. M. \& Kouwenhoven, L. P. Spin-orbit qubit in a semiconductor nanowire. Nature 468, 1084-1087 (2010).

8. Rashba, E. I. Properties of semiconductors with an extremum loop. 1. Cyclotron and combinational resonance in a magnetic field perpendicular to the plane of the loop. Sov. Phys. Solid State 2, 1109-1122 (1960).

9. Bychkov, Y. A. \& Rashba, E. I. Properties of a 2D electron gas with lifted spectral degeneracy. JETP Lett. 39, 78-81 (1984).

10. Xia, Y. et al. Observation of a large-gap topological-insulator class with a single Dirac cone on the surface. Nat. Phys. 5, 398-402 (2009).

11. Yazyev, O. V., Moore, J. E. \& Louie, S. G. Spin polarization and transport of surface states in the topological insulators $\mathrm{Bi} 2 \mathrm{Se} 3$ and $\mathrm{Bi} 2 \mathrm{Te} 3$ from first principles. Phys. Rev. Lett. 105, 266806 (2010).

12. Zhang, T. et al. Experimental demonstration of topological surface states protected by time-reversal symmetry. Phys. Rev. Lett. 103, 266803 (2009).

13. Roushan, P. et al. Topological surface states protected from backscattering by chiral spin texture. Nature 460, 1106-1109 (2009).

14. Schomerus, H. Helical scattering and valleytronics in bilayer graphene. Phys. Rev. B 82, 165409 (2010).

15. Gunlycke, D. \& White, C. T. Graphene valley filter using a line defect. Phys. Rev. Lett. 106, 136806 (2011).

16. Appelbaum, I., Huang, B. \& Monsma, D. J. Electronic measurement and control of spin transport in silicon. Nature 447, 295-298 (2007).

17. Goswani, S. et al. Controllable valley splitting in silicon quantum devices. Nat Phys. 3, 41-45 (2007).

18. Jansen, R. Silicon spintronics. Nat. Mat. 11, 400-408 (2012).

19. Oguchi, T. \& Shishidou, T. The surface Rashba effect: a $k \cdot p$ perturbation approach. J. Phys.: Condens. Matt. 21, 092001 (2009).

20. Sakamoto, K. et al. Abrupt rotation of the Rashba spin to the direction perpendicular to the surface. Phys. Rev. Lett. 102, 096805 (2009).

21. Ibañez-Azpiroz, J., Eiguren, A. \& Bergara, A. Relativistic effects and fully spin-polarized Fermi surface at the Tl/Si(111) surface. Phys. Rev. B 84, 125435 (2011).

22. Kocán, P., Sobotík, P. \& Ošt'ádal, I. Metallic-like thallium overlayer on a Si(111) surface. Phys. Rev. B 84, 233304 (2011).

23. Ohtsubo, Y., Hatta, S., Okuyama, H. \& Aruga, T. A metallic surface state with uniaxial spin polarization on $\mathrm{Tl} / \mathrm{Ge}(111)-(1 \times 1)$. J. Phys.: Condens. Matt. 24, 092001 (2012).

24. Zhou, S. Y. et al. First direct observation of Dirac fermions in graphite. Nat. Phys. 2, 595-599 (2006).

25. Rutter, G. M., Crain, J. N., Guisinger, N. P., Li, T., First, P. N. \& Stroscio, J. A. Scattering and interference in epitaxial graphene. Science 317, 219-222 (2006).

26. Mattet, P., Varchon, F., Naud, C., Magaud, L., Berger, C. \& Veuillen, J.-Y. Electron states of mono-and bilayer graphene on SiC probed by scanningtunneling microscopy. Phys. Rev. B 76, 041403 (2007).

27. Brihuega, I. et al. Quasiparticle chirality in epitaxial graphene probed at the nanometer scale. Phys. Rev. Lett. 101, 206802 (2008).

28. Okuda, T. et al. A new spin-polarized photoemission spectrometer with very high efficiency and energy resolution. Rev. Sci. Instrum. 79, 123117 (2008). 
29. Budke, M., Allmers, T., Donath, M. \& Rangelov, G. Combined experimental setup for spin- and angle-resolved direct and inverse photoemission. Rev. Sci. Instrum. 78, 113909 (2007).

30. Perdew, J. P. et al. Atoms, molecules, solids, and surfaces: Applications of the generalized gradient approximation for exchange and correlation. Phys. Rev. B 46, 6671-6687 (1992)

31. Hohenberg, P. \& Kohn, W. Inhomogeneous electron gas. Phys. Rev. 136, B864-B871 (1964).

32. Laasonen, K., Pasquarello, A., Car, R., Lee, C. \& Vanderbilt, D. Car-Parrinello molecular dynamics with Vanderbilt ultrasoft pseudopotentials. Phys. Rev. B 47, 10142-10153 (1993)

\section{Acknowledgements}

K.S. thanks T. Hirayama and K. Kobayashi for discussion. This research is supported by the Grant-in-Aid for Scientific Research (A) 20244045 and (B) 25287070, the Grant-inAid for JSPS Fellows and the G-COE programs (G-03). T.-H.K. and H.W.Y. are supported by the National Research Foundation of Korea through the Center for Low Dimensional Electronic Symmetry (Grant No. 2012R1A3A2026380) and the SRC Center for Topological Materials (Grant No. 2011-0030789).

\section{Author contributions}

K.S., T.K., B.M., Y.Y., J.O., R.U., K.M. and J.F. carried out the ARPES measurements. K.S., B.M., Y.Y., M.O., Y.T. and A.H. carried out SARPES. SARIPES experiments were carried out by S.D.S., K.S., A.B.S. and M.D. T.-H.K. and H.W.Y. performed the STM measurements. T.O. carried out the DFT calculation. K.S. analysed the data and wrote the manuscript with input from T.-H.K., T.K., B.M., J.O., R.U., Y.T., S.D.S., A.B.S., M.D., H.W.Y. and T.O. K.S. conceived and coordinated the project. All authors discussed the results and commented on the manuscript.

\section{Additional information}

Competing financial interests: The authors declare no competing financial interests.

Reprints and permission information is available online at http://npg.nature.com/ reprintsandpermissions/

How to cite this article: Sakamoto, K. et al. Valley spin polarization by using the extraordinary Rashba effect on silicon. Nat. Commun. 4:2073 doi: 10.1038/ncomms3073 (2013). 